\title{
Beobadhtung von Elementarprozessen an angeregten Molekülen
}

\author{
V. Dissoziation von Cyclohexan in Gleichstrom-, $50 \mathrm{~Hz}$ - und $535 \mathrm{~Hz}$-Entladungen \\ G. Arnold
}

Forschungsstelle für Spektroskopie in der Max-Planck-Gesellschaft, Göttingen

(Z. Naturforschg. 20 a, 435-441 [1965]; eingegangen am 14. Dezember 1964)

\begin{abstract}
Primary dissociation processes on excited cyclohexane have been investigated in d.c. and a.c. (50 cycles and 535 cycles) glow discharges. The $\mathrm{C}_{2}-\mathrm{C}_{3^{-}}$and $\mathrm{C}_{4}$-hydrocarbons formed, have been analysed by gaschromatography. These dissociation products can be explained quantitatively by a simple reaction scheme, which is based on $\mathrm{C}_{3}: \mathrm{C}_{3}, \mathrm{C}_{4}: \mathrm{C}_{2^{-}}$and $\mathrm{C}_{2}: \mathrm{C}_{2}: \mathrm{C}_{2}$-cleavages of the cyclohexane ring and the following intramolecular disproportionation of the biradicals formed. The composition of the dissociation products is more or less identical for the two different a.c. investigations. Different results are observed for a. c. and d. c. glow discharges.

The results indicate that the differences in the probabilities for the dissociation processes between a.c. and d.c. glow discharges are caused by the electrical property of the stationary and the moving striations of the positive column.
\end{abstract}

Im Rahmen der Strahlenchemie beschäftigen sich zahlreiche Arbeiten mit dem Zerfall von Kohlenwasserstoffen unter der Einwirkung energiereicher Strahlung ${ }^{1}$. Bei den dabei auftretenden chemischen Prozessen handelt es sich vorwiegend um Ion-Molekül-Reaktionen. In jüngster Zeit gewinnen jedoch Untersuchungen über den Einfluß niederenergetischer Strahlung auf den Zerfallsprozeß organischer Moleküle immer mehr an Bedeutung. Sie sind besonders dafür geeignet, einen Einblick in den Mechanismus der Dissoziationsvorgänge organischer Moleküle zu geben.

Wechselwirkungen von Elektronen und Lichtquanten geringer Energie mit Kohlenwasserstoffen führen hauptsächlich zu Dissoziationsprozessen angeregter neutraler Moleküle, wobei Molekül-Abspaltprozesse eine große Rolle spielen.

Elektronen niedriger Energie lassen sich leicht in der positiven Säule von Glimmentladungen erzeugen. In einer früheren Arbeit ${ }^{2}$ wurde eine Apparatur beschrieben, mit deren Hilfe den organischen Molekülen Energie von nur einigen Elektronenvolt zugeführt wird. Sie besteht im wesentlichen aus einem Entladungsrohr. Die zu untersuchenden Moleküle strömen kontinuierlich durch den Reaktionsraum. Die Aufenthaltsdauer beträgt 16 msec. Die Anregung der neutralen organischen Moleküle erfolgt durch Elektronenstoß in der positiven Säule der Glimmentladung. Die Energieverteilungsfunktion der Elek-

1 F. Williams, Quart. Rev. London 17, 101 [1963].

2 H. Schüler u. G. Arnold, Z. Naturforschg. 17 a, 670 [1962].

3 D. Barbière, Phys. Rev. 84, 653 [1951]. tronen zeigt in der positiven Säule einen scharfen Abfall in der Nähe der ersten Anregungsenergie des Füllgases ${ }^{3}$. Die Maximalenergie der Elektronen ist nahezu gleich dieser niedrigsten Anregungsenergie der in der Entladung am stärksten vorhandenen Substanz. Infolge dieser geringen Elektronenenergie werden in der positiven Säule kaum Ionen erzeugt ${ }^{4}$. Da außerdem die Beweglichkeit der Elektronen viel größer ist als die der Ionen, so wird der Strom in der positiven Säule praktisch nur von Elektronen getragen. Wie auch Kuppermans und Burton ${ }^{5}$ in ihrer Arbeit über den Zerfall von n-Butan bestätigten, sind Ion-Molekül-Reaktionen bei der Dissoziation organischer Moleküle in der positiven Säule einer Glimmentladung zu vernachlässigen.

Durch die kurze Aufenthaltsdauer eines Moleküls im Reaktionsraum kann unter den gegebenen Versuchsbedingungen ( $10^{19}$ Moleküle werden pro sec. in den Reaktionsraum hineinverdampft, dem in der gleichen Zeit eine Energie von $10^{17} \mathrm{eV}$ zugeführt wird) ein Molekül nur einmal angeregt werden. Da weiterhin bei einem Substanzdruck von ca. 2 Torr im Reaktionsraum die mittlere Zeit zwischen zwei Zusammenstößen $10^{-7}$ sec beträgt und ein angeregtes Molekül eine maximale Lebensdauer von etwa $10^{-8}$ sec besitzt (Triplett-Übergänge ausgenommen), können mit der in ${ }^{2}$ ausführlich beschriebenen Methode primäre Dissoziationsprozesse an isolierten, angeregten Molekülen untersucht werden.

4 E. J. Lauer, J. Appl. Phys. 23, 300 [1952].

5 A. Kuppermann u. M. Burton, Radiation Res. 10, 636 [1959]. 
Bei den bisherigen Untersuchungen ${ }^{2,6,7}$ wurden primäre Elementarprozesse beim Zerfall von angeregtem Cyclohexan und n-Butan beobachtet. Zur Deutung der dabei auftretenden Dissoziationsprodukte wurden einfache Reaktionsschemata aufgestellt, die sich quantitativ bestätigen. Bei diesen Versuchen wurde die Entladungsröhre mit $50 \mathrm{~Hz}$ Wechselstrom betrieben. Für n-Butan ${ }^{8}$ konnte gezeigt werden, daß die Dissoziationsprozesse in $50 \mathrm{~Hz}$ und Gleichstrom-Entladungen verschieden sind. Es wurden zwar auch bei den Gleichstromuntersuchungen primäre Elementarprozesse beobachtet, jedoch unterschieden sich die relativen Häufigkeiten der Zerfallsreaktionen von denen bei Wechselstromuntersuchungen um ganzzahlige Vielfache. Dieser Effekt wird auch beim Cyclohexan beobachtet und soll in der vorliegenden Arbeit näher untersucht werden. Darüber hinaus wird auch über Versuche mit $535 \mathrm{~Hz}$ Wechselstrom berichtet, die über den Frequenzeinfluß Auskunft geben sollen.

\section{Resultate}

Wie in den früheren Arbeiten, wurden wieder die gasförmigen Reaktionsprodukte des Cyclohexanzerfalls $\left(\mathrm{C}_{2^{-}}, \mathrm{C}_{3}-\mathrm{C}_{4}\right.$-Kohlenwasserstoffe $)$ quantitativ untersucht. Hierzu wurde das mit flüssiger Luft aus der Entladung in einem Auffanggefäß kondensierte Gemisch aus unzersetztem Cyclohexan und Dissoziationsprodukten gaschromatographisch analysiert (4 m-Hexadekan-Kolonne mit 30-proz. Belegung; 3,60 m Di-n-Butylmaleat-Kolonne mit 30-proz. Belegung; 1,80 m Hexadekan-Kolonne mit 2-proz. Belegung; Flammenionisationsdetektor).

In Abb. 1 ist die Zahl der pro sec dissoziierten Cyclohexanmoleküle $n$, bezogen auf die pro sec zugeführten Cyclohexanmoleküle $n_{0}$, als Funktion der Entladungsstromstärke für die 3 verschiedenen Stromarten dargestellt. Diese wurde aus den gaschromatographisch gemessenen Mengen $\operatorname{der} \mathrm{C}_{2}{ }^{-}, \mathrm{C}_{3}$. und $\mathrm{C}_{4}$-Reaktionsprodukte unter der Annahme berechnet, daß letztere aus $\mathrm{C}_{3}: \mathrm{C}_{3}{ }^{-}, \mathrm{C}_{4}: \mathrm{C}_{2^{-}}$und $\mathrm{C}_{2}: \mathrm{C}_{2}: \mathrm{C}_{2}$-Trennungen des Cyclohexanringes hervorgehen. Da die Sondenspannung an der positiven Säule für jede Stromart jeweils konstant war, folgt aus der linearen Abhängigkeit $n / n_{0}=\alpha \cdot I$, da $n / n_{0}$ auch mit der Leistung der Entladung linear ansteigt.

${ }^{6}$ H. Schüler u. G. Arnold, Z. Naturforschg. 18 a, 15 [1963].

7 H. Schüler u. G. Arnold, Z. Naturforschg. 18 a, 604 [1963].
Die beobachteten Reaktionsprodukte entstehen also aus dem angeregten Cyclohexan durch primäre Dissoziationsprozesse.

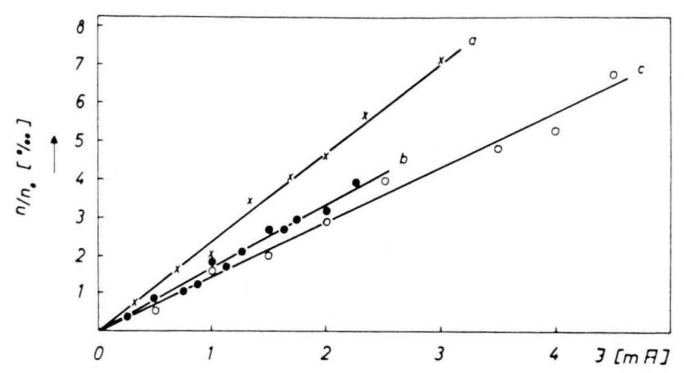

Abb. 1. Die pro sec in $\mathrm{C}_{2}-\mathrm{C}_{3}$ - und $\mathrm{C}_{4}$-Kohlenwasserstoffe zerfallenen Cyclohexanmoleküle $n$, bezogen auf die pro sec zugeführten Cyclohexanmoleküle $n_{0}$, in Abhängigkeit von der Entladungsstromstärke. a: $50 \mathrm{~Hz}$-Entladung $(30 \mathrm{~V} / \mathrm{cm})$, b: Gleichstromentladung $(41 \mathrm{~V} / \mathrm{cm})$, c: $535 \mathrm{~Hz}$-Entladung $(20 \mathrm{~V} / \mathrm{cm})$.

\section{$\mathrm{C}_{3}: \mathrm{C}_{3}$-Trennung}

Es wurden sechs $\mathrm{C}_{3}$-Reaktionsprodukte gaschromatographisch nachgewiesen, deren prozentuale Anteile, bezogen auf alle gebildeten $\mathrm{C}_{3}$-Kohlenwasserstoffe, in den Abb. $2 \mathrm{a}-\mathrm{c}$ für die 3 verschie-
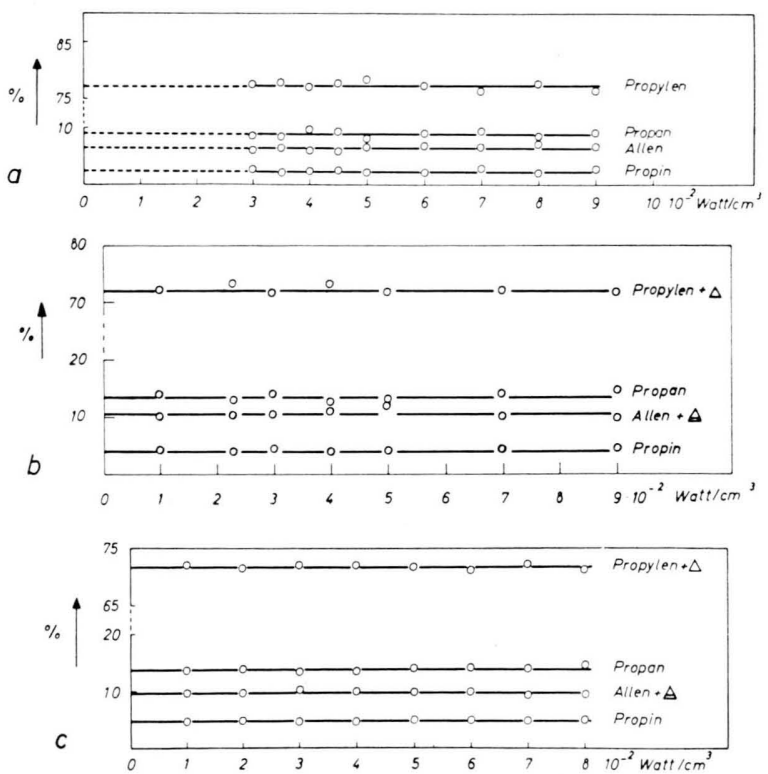

Abb. 2. Relative Ausbeuten der bei der Dissoziation des Cyclohexans entstehenden $\mathrm{C}_{3}$-Reaktionsprodukte als Funktion der Leistung im Reaktionsraum. a: Gleichstromentladung, b: $50 \mathrm{~Hz}$-Entladung, c: $535 \mathrm{~Hz}$-Entladung. $\triangle$ : Cyclopropan, $\triangle$ : Cyclopropylen.

8 H. Schüler u. G. Arnold, Z. Naturforschg. 19 a, 771 [1964]. 
denen Stromarten als Funktion der Leistung im Reaktionsraum dargestellt sind. In allen 3 Fällen sind die relativen Ausbeuten der $\mathrm{C}_{3}$-Reaktionsprodukte von der Leistung der Entladung unabhängig.

Für die Gleichstromuntersuchungen sind diese relativen Ausbeuten nur für Leistungen $\geqq 3 \cdot 10^{-2}$ Watt $/ \mathrm{cm}^{3}$ dargestellt. Bei kleineren Leistungen wird die Gleichstromentladung durch hochfrequente Schwingungen $\left(\geqq 10^{5} \mathrm{~Hz}\right)$ der Spannung gestört, deren Amplituden ca. 5-10\% der Gleichspannung betragen. War bisher die Sondenspannung praktisch von der Stromstärke unabhängig, so sinkt unterhalb von $3 \cdot 10^{-2} \mathrm{Watt} / \mathrm{cm}^{3}$ die Spannung mit abnehmender Stromstärke. Die Änderung der elektrischen Entladungseigenschaften hat auch eine Änderung in der Zusammensetzung der Reaktionsprodukte zur Folge. Unterhalb von $3 \cdot 10^{-2} \mathrm{Watt} / \mathrm{cm}^{3}$ werden die relativen Ausbeuten der $\mathrm{C}_{3}$-Reaktionsprodukte mit sinkender Leistung den Wechselstromwerten ähnlicher. Wechselstrom- und Gleichstromeffekte werden wahrscheinlich mit unterschiedlichen Gewichten überlagert.

Die aus den Abb. 2 a - c folgenden Werte sind in Tab. 1 zusammengestellt. Wie in der ersten Arbeit ${ }^{2}$ bereits ausführlich gezeigt wurde, lassen sich diese $\mathrm{C}_{3}$-Zerfallsprodukte durch das in Abb. 3 wiedergegebene Reaktionsschema erklären.

\begin{tabular}{|l|r|r|r|}
\hline & Gleich- & \multicolumn{2}{|c|}{ Wechselstrom } \\
& strom & $50 \mathrm{~Hz}$ & $535 \mathrm{~Hz}$ \\
\hline Propylen $+\triangle$ & $82,4 \%$ & $72,1 \%$ & $71,7 \%$ \\
Propan & $8,9 \%$ & $13,5 \%$ & $13,8 \%$ \\
Propin & $2,3 \%$ & $4,0 \%$ & $4,8 \%$ \\
Allen $+\triangle$ & $6,4 \%$ & $10,4 \%$ & $9,7 \%$ \\
\hline
\end{tabular}

Tab. 1.

Und zwar lassen sich die experimentellen Werte der Tab. 1 aus den Primärprozessen der Abb. 3 quantitativ erklären, wenn für die Prozesse $3 \alpha-3 \gamma$ die in Tab. 2 angegebenen ganzzahligen Gewichte angenommen werden.

\begin{tabular}{|c|c|c|c|c|}
\hline & $3 \alpha 3 \beta 3 \gamma$ & $\mathrm{C}_{3} \mathrm{H}_{6}$ & $\mathrm{C}_{3} \mathrm{H}_{8}$ & $\mathrm{C}_{3} \mathrm{H}_{4}$ \\
\hline $\begin{array}{l}\text { Gleichstrom } \\
50 \mathrm{~Hz} \text { - }\end{array}$ & $\begin{array}{lll}7 & 2 & 2\end{array}$ & $81,8 \%$ & $9,1 \%$ & $9,1 \%$ \\
\hline $\begin{array}{l}\text { Wechselstrom } \\
535 \mathrm{~Hz} \text { - } \\
\text { Wechselstrom }\end{array}$ & $\begin{array}{lll}3 & 2 & 2 \\
& & \\
3 & 2 & 2\end{array}$ & $71,4 \%$ & $14,3 \%$ & $14,3 \%$ \\
\hline
\end{tabular}

Tab. 2.

9 S. W. Benson, J. Amer. Chem. Soc. 84, 3411 [1962].

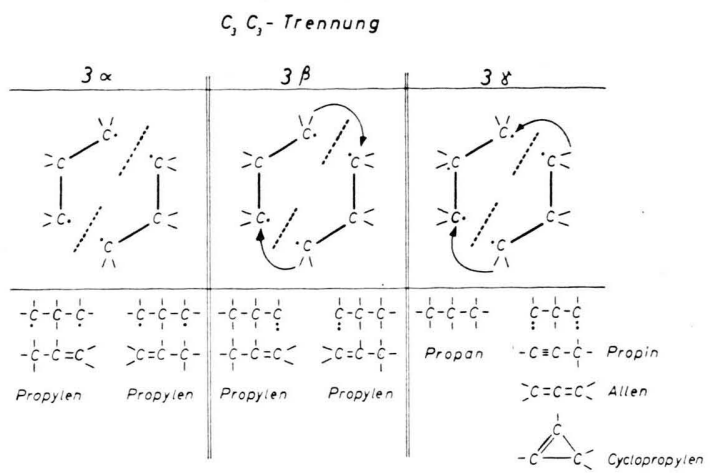

Abb. 3. Reaktionsschema zur quantitativen Deutung der beobachteten $\mathrm{C}_{3}$-Zerfallsprodukte durch Primärprozesse.

Durch eine $\mathrm{C}_{3}: \mathrm{C}_{3}$-Trennung des Cyclohexanringes entstehen zunächst zwei Biradikale. Nach Benson ${ }^{9}$ ist die Lebensdauer dieser Biradikale von der Größenordnung von $10^{-10}-10^{-11}$ sec, so daß Stoßprozesse für die Stabilisierung der Biradikale zu vernachlässigen sind. Die intermediär auftretenden Biradikale stabilisieren sich durch Bildung einer Doppelbindung bzw. Dreifachbindung oder durch intramolekulare Disproportionierung ( $\mathrm{H}-\mathrm{W}$ anderung im Falle $3 \gamma$ ). Es handelt sich hierbei also um intramolekulare Prozesse der Molekülabspaltung, die auch von Srinivasan ${ }^{10}$ bei dem Zerfall des Cyclohexadiens angenommen wurden.

Bei den Gleichstromuntersuchungen ist weder Cyclopropan noch Cyclopropylen meßbar. Das zum Propylen hinzugezählte Cyclopropan beträgt bei den Wechselstromuntersuchungen etwa $0,6 \%$. Es wird wahrscheinlich durch Ringschluß aus dem Biradikal $\dot{\mathrm{C}} \mathrm{H}_{2}-\mathrm{CH}_{2}-\dot{\mathrm{C}} \mathrm{H}_{2}$ gebildet. Für $50 \mathrm{~Hz}$ und $535 \mathrm{~Hz}$ folgt für das Verhältnis von Allen/Cyclopropylen $=0,9$.

Innerhalb der Fehlergrenzen stimmen die Ergebnisse für die $50 \mathrm{~Hz}$ - und $535 \mathrm{~Hz}$-Untersuchungen überein. Eventuelle Unterschiede, die bei den Gleichstromergebnissen besonders deutlich werden, zeichnen sich bei der Stabilisierung des $\dot{\mathrm{C}} \mathrm{H}-\mathrm{CH}_{2}-\dot{\mathrm{C}} \mathrm{H}$ Biradikals ab. Bei den $535 \mathrm{~Hz}$-Versuchen tritt Propin und Allen zu gleichen Teilen auf, dagegen wird bei den Gleichstromversuchen dreimal mehr Allen als Propin gebildet.

\section{$\mathrm{C}_{4}: \mathrm{C}_{2}$-Trennung}

In Abb. $4 \mathrm{a}-\mathrm{c}$ sind die beim Cyclohexanzerfall beobachteten $\mathrm{C}_{4}$-Reaktionsprodukte analog zu den

10 R. Srinivasan, J. Chem. Phys. 38, 1039 [1963]. 
$\mathrm{C}_{3}$-Kohlenwasserstoffen dargestellt. Als Ordinate sind die Ausbeuten der verschiedenen $\mathrm{C}_{4}$-Reaktionsprodukte als prozentuale Anteile der gesamten $\mathrm{C}_{4}$. Produkte aufgetragen. Für alle 3 Stromarten sind diese relativen Ausbeuten von der Leistung im Reaktionsraum unabhängig. Diese Konstanz ist zusammen mit den in Abb. 1 dargestellten Befunden eine Bestätigung dafür, daß die beobachteten Dissoziationsprodukte durch primäre Elementarprozesse aus dem angeregten Cyclohexan gebildet werden. Die aus den Abb. $4 \mathrm{a}-\mathrm{c}$ folgenden relativen Ausbeuten sind in Tab. 3 zusammengestellt.

\begin{tabular}{|l|r|r|r|}
\hline & \multicolumn{2}{|c}{$\begin{array}{c}\text { Gleich- } \\
\text { strom }\end{array}$} & \multicolumn{2}{c|}{ Wechselstrom } \\
& & $50 \mathrm{~Hz}$ & $535 \mathrm{~Hz}$ \\
\hline Butadien & $34,7 \%$ & $36,3 \%$ & $32,4 \%$ \\
Buten-1 & $8,5 \%$ & $24,5 \%$ & $22,4 \%$ \\
trans-Buten-2 & $3,3 \%$ & $15,9 \%$ & $18,7 \%$ \\
cis-Buten-2 & $1,7 \%$ & $7,2 \%$ & $8,2 \%$ \\
iso-Buten & - & $2,0 \%$ & $2,5 \%$ \\
iso-Butan & - & $3,8 \%$ & $4,9 \%$ \\
n-Butan & $1,8 \%$ & $10,3 \%$ & $11,0 \%$ \\
\hline
\end{tabular}

Tab. 3 .

In Abb. 4 a sind wieder wie in Abb. 2 a die rela tiven Ausbeuten der $\mathrm{C}_{4}$-Reaktionsprodukte nur für den Leistungsbereich $\geqq 3 \cdot 10^{-2} \mathrm{~W}$ att $/ \mathrm{cm}^{3}$, in dem eine ungestörte Gleichstromentladung existiert, dar-
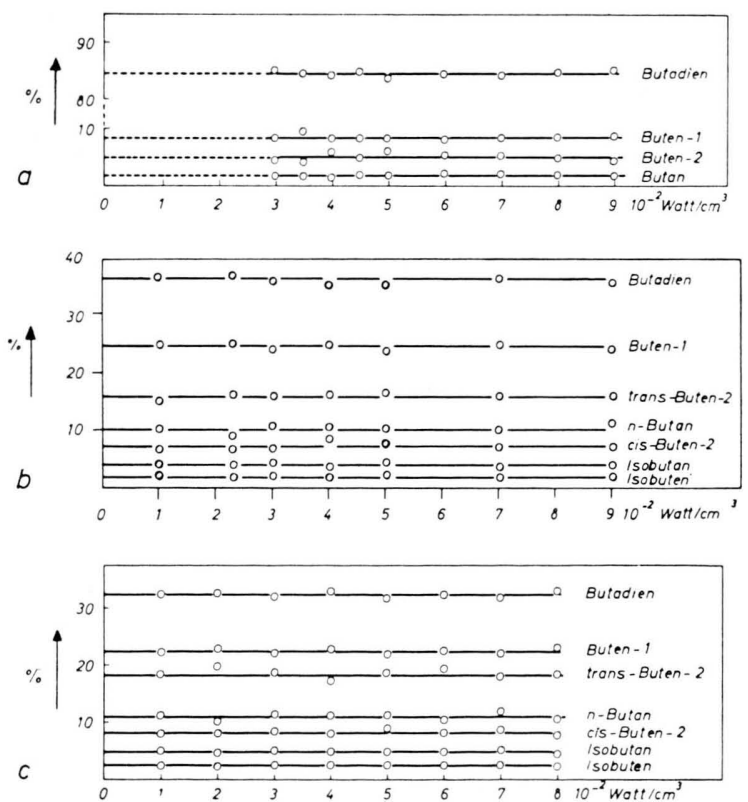

Abb. 4. Relative Ausbeuten der bei der Dissoziation des Cyclohexans entstehenden $\mathrm{C}_{4}$-Reaktionsprodukte als Funktion der Leistung im Reaktionsraum. a: Gleichstromentladung, b: $50 \mathrm{~Hz}$-Entladung, c: $535 \mathrm{~Hz}$-Entladung. gestellt. Zur Deutung der beobachteten Dissoziationsprodukte soll zunächst ein der Abb. 3 entsprechendes Reaktionsschema zugrunde gelegt werden. Es ergeben sich dann die in Abb. 5 dargestellten Bildungs. möglichkeiten. Neben der alleinigen Trennung zweier C - C-Bindungen $(5 \alpha)$ kann noch zwischen 3 Prozessen $(5 \beta-5 \delta)$ mit der Trennung zweier $\mathrm{C}-\mathrm{C}$-Bindungen und ungleichen intramolekularen $\mathrm{H}$-Wanderungen unterschieden werden. Infolge dieser unterschiedlichen intramolekularen Disproportionierungsmöglichkeiten werden diese 4 Prozesse im allgemeinen nicht gleichwahrscheinlich sein.

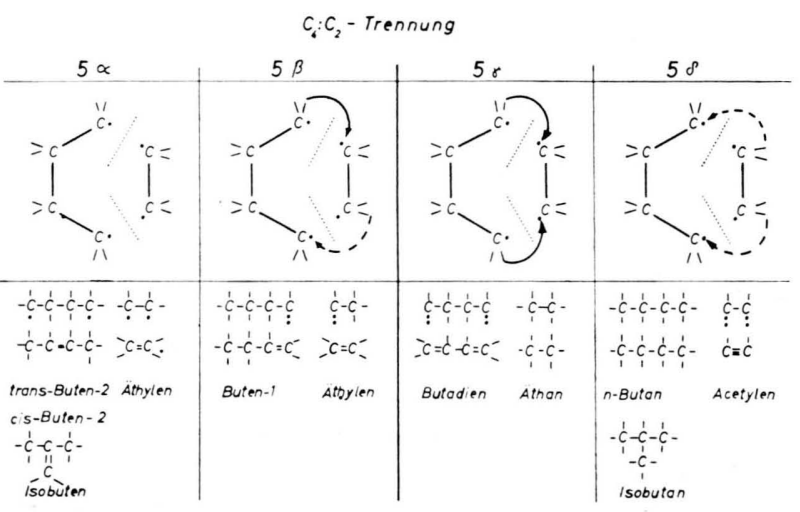

Abb. 5. Reaktionsschema zur quantitativen Deutung der beobachteten $\mathrm{C}_{4}$-Zerfallsprodukte durch Primärprozesse.

Für Wechselstrom ergibt sich experimentell für die Verhältnisse Butadien/Äthan $=1,08$ und für Bu$\tan /$ Acetylen $=0,9 ;$ das entspricht etwa dem aus $5 \gamma$ und $5 \delta$ erwarteten Wert von 1,0. Aus den Wechselstromergebnissen der Tab. 3 können dann für die Prozesse $5 \alpha-5 \delta$ die in Tab. 4 angegebenen Gewichte, mit denen sie auftreten, errechnet werden.

\begin{tabular}{|c|r|c|c|c|c|c|}
\hline \multirow{2}{*}{$\begin{array}{c}\text { Pro- } \\
\text { zeß }\end{array}$} & Gew. & \multicolumn{2}{|c|}{$\begin{array}{c}50 \mathrm{~Hz} \\
\text { relat. Häufigkeit }\end{array}$} & \multicolumn{3}{c|}{$\begin{array}{c}535 \mathrm{~Hz} \\
\text { relat. Häufigkeit }\end{array}$} \\
\hline 5 & 7 & $25,0 \%$ & $25,1 \%$ & 4 & $30,8 \%$ & $29,4 \%$ \\
$5 \alpha$ & 7 & $25,0 \%$ & $24,5 \%$ & 3 & $23,1 \%$ & $22,4 \%$ \\
$5 \beta$ & 10 & $35,7 \%$ & $36,3 \%$ & 4 & $30,8 \%$ & $32,3 \%$ \\
$5 \gamma$ & 10 & gemessen & Gew. & berechn. & gemessen \\
$5 \delta$ & 4 & $14,3 \%$ & $14,1 \%$ & 2 & $15,4 \%$ & $15,9 \%$ \\
\hline
\end{tabular}

Tab. 4.

Bei dem Cyclohexanzerfall in der Gleichstromentladung tritt unter den $\mathrm{C}_{4}$-Reaktionsprodukten Butadien am stärksten auf. Aus dem Verhältnis Butadien/Äthan $\gg 1$ folgt, daß für die Butadienbildung neben dem Prozeß $5 \gamma$ noch eine andere Möglichkeit existieren muß. Diese starke Butadien- 
bildung kann durch nachfolgenden Primärprozeß erklärt werden :

$$
\text { Cyclo- } \mathrm{C}_{6} \mathrm{H}_{12}{ }^{*} \rightarrow \mathrm{C}_{4} \mathrm{H}_{6}+\mathrm{C}_{2} \mathrm{H}_{4}+\mathrm{H}_{2} \text {. }
$$

Die dem Cyclohexanmolekül durch Elektronenstoß zugeführte Energie (die Cyclohexananregung liegt bei etwa $7,3 \mathrm{eV}$ entsprechend $168 \mathrm{kcal}$ ) reicht nur zur Trennung zweier C-C-Bindungen aus. Die zur $\mathrm{H}_{2}$-Abspaltung notwendige Energie wird aus der Stabilisierung der Biradikale, die bei einer $\mathrm{C}_{4}: \mathrm{C}_{2}$ Trennung des Cyclohexanringes entstehen, unter Ausbildung von Doppelbindungen gewonnen.

Wahrscheinlich verläuft der Prozeß (1) parallel zu den Prozessen $5 \alpha-5 \delta$, denn die restlichen $\mathrm{C}_{4}$. Reaktionsprodukte (Buten-1, Buten-2, Butan) können nach $5 \alpha, 5 \beta$ und $5 \delta$ erklärt werden. Diese 3 Prozesse verhalten sich in der Gleichstromentladung in dieser Reihenfolge wie $5: 3: 1$.

$$
\mathrm{C}_{2}: \mathrm{C}_{2}: \mathrm{C}_{2} \text {-Trennung }
$$

Ein Vergleich zwischen der pro Sekunde gebildeten Menge von $\mathrm{C}_{2}$ - und $\mathrm{C}_{4}$-Kohlenwasserstoffen ergibt, daß noch ein nur Äthylen liefernder Prozeß existieren muß. In Analogie zu dem von SrinivaSAN ${ }^{10}$ erwähnten intramolekularen Zerfall des Cyclohexadiens:

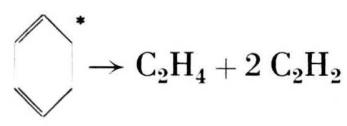

kann das überschüssige Äthylen beim CyclohexanZerfall durch folgenden Primärprozeß erklärt werden:

$$
\text { Cyclo- } \mathrm{C}_{6} \mathrm{H}_{12}{ }^{*} \rightarrow 3 \mathrm{C}_{2} \mathrm{H}_{4} \text {. }
$$

Die Energie für die dritte C - C-Trennung wird hierbei wieder aus der Stabilisierung der Biradikale gewonnen, die bei der Trennung der ersten beiden $\mathrm{C}$ - C-Bindungen entstehen. Es ist die gleiche Energie, die in Prozeß (1) zur $\mathrm{H}_{2}$-Abspaltung führte.

Die Gesamtheit der beobachteten $\mathrm{C}_{2}-\mathrm{C}_{3}$ - und $\mathrm{C}_{4}$ Dissoziationsprodukte des angeregten Cyclohexans läßt sich somit durch die Primärprozesse (1) und (2), sowie durch die Primärprozesse der in Abb. 3 und Abb. 5 dargestellten Reaktionschemata erklären. Die relativen Häufigkeiten der Trennungsprozesse sind von der Leistung der Entladung unabhängig. Die Mittelwerte dieser relativen Häufigkeiten sind

\begin{tabular}{|c|c|c|c|c|c|}
\hline & \multirow{2}{*}{$\begin{array}{l}\text { Gleich- } \\
\text { strom }\end{array}$} & \multicolumn{2}{|c|}{ Wechselstrom } & \multirow{2}{*}{ 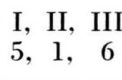 } & \multirow{2}{*}{$\begin{array}{l}\text { I, II, III } \\
4,3,3\end{array}$} \\
\hline & & $50 \mathrm{~Hz}$ & $535 \mathrm{~Hz}$ & & \\
\hline$C_{2}$ & $76,4 \%$ & $56,9 \%$ & $55,8 \%$ & $76,6 \%$ & $56,5 \%$ \\
\hline $\mathrm{C}_{3}$ & $6,4 \%$ & $25,5 \%$ & $27,1 \%$ & $6,7 \%$ & $26,1 \%$ \\
\hline $\mathrm{C}_{4}$ & $17,2 \%$ & $17,6 \%$ & $17,1 \%$ & $16,7 \%$ & $17,4 \%$ \\
\hline $\mathrm{C}_{4}: \mathrm{C}_{2}$ & $42,7 \%$ & $40,6 \%$ & $39,4 \%$ & $41,7 \%$ & $40,0 \%$ \\
\hline $\mathrm{C}_{3}: \mathrm{C}_{3}$ & $8,0 \%$ & $29,2 \%$ & $31,0 \%$ & $8,3 \%$ & $30,0 \%$ \\
\hline $\mathrm{C}_{2}: \mathrm{C}_{2}: \mathrm{C}_{2}$ & $49,3 \%$ & $30,2 \%$ & $29,6 \%$ & $50,0 \%$ & $30,0 \%$ \\
\hline
\end{tabular}
für die 3 Stromarten in Tab. 5 zusammengestellt $\left(\mathrm{C}_{2}+\mathrm{C}_{3}+\mathrm{C}_{4}=100 \%\right.$ und $\left.\mathrm{I}+\mathrm{II}+\mathrm{III}=100 \%\right)$.
Tab. 5.

Nimmt man für die einzelnen Trennungsprozesse $\mathrm{I}=5 \alpha+5 \beta+5 \gamma+5 \delta+(1) ; \quad \mathrm{II}=3 \alpha+3 \beta+3 \gamma ;$ $\mathrm{III}=(2)$ die in Tab. 5 , Spalte 5 und 6 oben, angegebenen Gewichte an, so lassen sich für die $\mathrm{Zu}$ sammensetzungen der Reaktionsprodukte die darunter stehenden Werte berechnen.

\section{Diskussion}

Wie bei den Untersuchungen am n-Butan ${ }^{8}$, läßt sich die Bildung der beobachteten Dissoziationsprodukte des angeregten Cyclohexans sowohl für Wechsel- als auch für Gleichstrom durch Primärprozesse erklären, obwohl die relativen Häufigkeiten der einzelnen Primärprozesse zwischen Gleich- und Wechselstrom sehr verschieden sind.

In der Gleichstromentladung wird bei einer $\mathrm{C}_{3}: \mathrm{C}_{3}$ Trennung des Cyclohexanringes die Trennung ohne Disproportionierung mit anschließender intramolekularer Stabilisierung der beiden Biradikale bevorzugt. Auch bei dem $\mathrm{C}_{4}: \mathrm{C}_{2}$-Zerfall wird die Trennung ohne Disproportionierung bevorzugt, der jedoch noch eine $\mathrm{H}_{2}$-Abspaltung am $\mathrm{C}_{4}$-Bruchstück folgt. Dadurch wird die intramolekulare Disproportionierung (Prozesse $5 \beta, 5 \gamma, 5 \delta$ ) stark unterdrückt. Parallel zu diesem Prozeß (1) der $\mathrm{H}_{2}$-Abspaltung läuft der Prozeß (2), so daß in der Gleichstromentladung die $\mathrm{C}_{2}: \mathrm{C}_{2}: \mathrm{C}_{2}$-Trennung verhältnismäßig stark auftritt. Die Ergebnisse des Cyclohexan-Zerfalls in der $50 \mathrm{~Hz}-$ und $535 \mathrm{~Hz}$-Entladung entsprechen dagegen mehr einer Gleichwahrscheinlichkeit der einzelnen Primärprozesse. Die relativen Häufigkeiten der Prozesse I, II und III sowie der Prozesse $3 \alpha-3 \gamma$ und $5 \alpha-5 \delta$ unterscheiden sich für die beiden Frequenzen nicht wesentlich. Einige Versuche mit $25 \mathrm{~Hz}$-Wechselstrom lieferten die gleichen Ergebnisse wie die $50 \mathrm{~Hz}$ Untersuchungen. Es wird praktisch keine Frequenzabhängigkeit der relativen Häufigkeiten der primären Dissoziationsprozesse beim Úbergang von $535 \mathrm{~Hz}$ über $50 \mathrm{~Hz}$ zu $25 \mathrm{~Hz}$ beobachtet; dagegen ändern 
sich diese relativen Häufigkeiten sprunghaft beim Übergang von Wechselstrom- zu Gleichstromentladungen.

Aus der Tatsache, daß zur quantitativen Erklärung der Dissoziationsprodukte keine intermolekularen Wechselwirkungen angenommen werden müssen, folgt für den Ablauf eines geschlossenen Dissoziationsprozesses eine Zeit, die kleiner ist als die mittlere Zeit zwischen zwei Zusammenstößen von $10^{-7}$ sec. Die Frequenzen des Entladungsstromes von $50 \mathrm{~Hz}$ und $535 \mathrm{~Hz}$ können somit für die unterschiedlichen Befunde der Wechselstrom- und Gleichstromentladungen nicht verantwortlich sein. Der Grund muß in der Eigenart der Entladung liegen.

\section{Eigenschaften der positiven Säule einer Glimmentladung}

Es existieren 3 Erscheinungsformen der positiven Säule: die homogene Säule und die Säule mit stationären bzw. mit wandernden Schichten. In der homogenen Säule der Glimmentladung sind in der Längsrichtung Elektronenenergie, Potentialbfall und Lichtemission örtlich und zeitlich konstant. Die homogene Säule tritt besonders bei reinen Edelgasen und niedrigem Druck ( $<1$ Torr) auf ${ }^{11}$. In der Regel werden aber in der positiven Säule von Entladungen - auch im Falle des Gleichstroms - Schichtund Schwingungserscheinungen beobachtet ${ }^{12}$, die nach Pfau und Rutscher ${ }^{13}$ den Prototyp der Niederdrucksäule darstellen. In der geschichteten Säule treten in der Längsrichtung periodisch helle und dunkle Zonen auf. Die hellen Zonen sind Gebiete maximalen Potentialabfalles. Die stationären Schichten sind typisch für Entladungen in molekularen Gasen bei Drucken von >1 Torr. Laufende Schichten treten zwar hauptsächlich in Edelgasen auf, werden unter bestimmten Bedingungen aber auch in molekularen Gasen beobachtet. Die Schichten bewegen sich mit Geschwindigkeiten von $10^{2}-10^{5} \mathrm{~cm}$ pro sec. Die Oszillationen sind eine Eigenschaft des Plasmas der Säule und hängen einerseits von der Stromstärke, dem Gasdruck, dem Rohrdurchmesser und der Natur des Gases ab und sind andererseits eng mit den Phänomenen in der Umgebung der

11 G. Francis, Handbuch der Physik, Bd. XXII, Springer-Verlag, Berlin 1956 II, S. $114 \mathrm{f}$.

12 G. Lakatos u. J. Bató, Acta Phys. Acad. Sci. Hung. 13, 193 [1961].

13 S. Pfau u. A. Rutscher, Ann. Phys., Lpz. 6, 244 [1960].
Elektroden gekoppelt ${ }^{14}$. Wie bereits erwähnt, wurden bei den vorliegenden Gleichstromuntersuchungen Schwingungen von $\geqq 10^{5} \mathrm{~Hz}$ beobachtet. Die Frequenzen dieser Plasma-Oszillationen können allgemein zwischen $10^{4}$ und $10^{9} \mathrm{~Hz}$ liegen. Sie ändern sich diskontinuierlich mit der Stromstärke ${ }^{11,12}$.

Laufende und stehende Schichten können künstlich durch eine Störung in einem vorher homogenen Säulenstück erzeugt werden ${ }^{13}$. Eine solche Störung stellt z. B. die in die Säule hineinragende Sonde zur Spannungsmessung oder ein Rohransatz dar. Die vorliegenden Untersuchungen über den CyclohexanZerfall wurden mit einem Entladungsrohr durchgeführt, das derartige Störstellen besitzt; diese wurden eingehend von Fowler ${ }^{15}$ untersucht. Er zeigte, $\mathrm{da} ß$ in einem Hauptentladungsrohr mit Seitenstutzen in dem Entladungsraum, welcher der Öffnung des Seitenstutzens entspricht, nie Schichten auftreten, wenn auch sonst die ganze positive Säule Schichtungen zeigt. Wandern die Schichten im Entladungs. rohr kontinuierlich, so springen die Schichten über die Strecke des Rohransatzes. Der Seitenstutzen vergrößert die effektive Begrenzung des Entladungs. rohres, so da $ß$ auf dieser Strecke die Bedingung für die Existenz von Schichten nicht erfüllt ist.

\section{Einfluß der geschichteten positiven Säule auf die Dissoziationsprozesse}

Die Untersuchungen über die Dissoziation angeregten Cyclohexans in der Glimmentladung wurden mit dem SchüLerschen Entladungsrohr durchgeführt. Bei Wechselstromentladungen durch organische Dämpfe bei einem Druck von $1-3$ Torr konnten Schüler ${ }^{16}$ und Michel ${ }^{17}$ in dieser Apparatur Schichtungen der positiven Säule nachweisen. Sie zeigten, daß in diesen Schichten Kondensationserscheinungen auftreten können. Und zwar handelt es sich dabei um durch Dissoziation bzw. Polymerisation entstandene Reaktionsprodukte. Das ist ein Hinweis darauf, daß die Schichtungen die Dissoziations. prozesse beeinflussen können.

Bei den vorliegenden Gleichstromuntersuchungen am Cyclohexan und bei den Gleichstromuntersuchungen am n-Butan ${ }^{8}$ läßt sich unterhalb einer gewissen

14 M. Klerk, Proc. 4th Intern. Conf. on Ionization Phenomena in Gases, Uppsala 1959, S. 283.

15 R. G. Fowler, Phys. Rev. 84, 145 [1951].

16 H. Schüler, J. Phys. Radium 15, 524 [1954].

17 A. Michel, Z. Naturforschg. 9 a, 788 [1954]. 
Leistung keine ungestörte Gleichstromentladung mehr einstellen. Die Sondenspannung ändert sich sprunghaft. Man beobachtet entweder ein Springen zwischen zwei Werten, bis sich der neue Wert einstellt oder der Entladungszustand bleibt instabil. Dieser Befund entspricht der diskontinuierlichen Änderung der Oszillationsfrequenz der Schichten in der positiven Säule. Die Änderung der Schichtung und damit die Änderung der elektrischen Eigenschaften bei kleinen Gleichstromleistungen wirken sich sehr stark auf die Dissoziationsprozesse aus. Die Ergebnisse bei Gleichstromentladungen geringer Leistung entsprechen bei den n-Butan-Versuchen den Wechselstromwerten und bei den vorliegenden Cyclohexan-Versuchen nähern sie sich stark den Wechselstromwerten. Daraus läßt sich vermuten, daß die Unterschiede zwischen den Wechselstrom- und Gleichstromergebnissen durch unterschiedliche Schichtungen der positiven Säule hervorgerufen werden. Auch über die Art der Beeinflussung lassen sich vorläufig nur Vermutungen äußern. Wahrscheinlich treten bei der Gleichstrom. entladung stationäre Schichten und bei der Wechselstromentladung laufende Schichten auf.

Wie bereits erwähnt, wird bei den Gleichstromentladungen geringer Leistung die konstante Entladungsspannung von schwachen hochfrequenten Schwingungen überlagert. Diese Schwingungen kön-

18 G. D. Morgan, Nature, Lond. 172, 542 [1953]. nen eine schwach variierende Elektronendichte zur Folge haben. Nach Morgan ${ }^{18}$ ruft eine am Ende der Plasmasäule vorhandene variierende Elektronendichte aber eine laufende Welle der Elektronendichte und damit eine laufende Schichtung der positiven Säule hervor. Mit einer am Säulenende konstanten Elektronendichte sind stehende Wellen und damit stationäre Schichten verbunden. Bei einer ungestörten Gleichstromentladung wird man also eine stationäre Schichtung erwarten. Das heißt im Falle der Gleichstromentladung existiert nach den Befunden von Fowler ${ }^{15}$ im Reaktionsraum eine konstante Potentialverteilung; dagegen tritt bei der Wechselstromentladung eine sprunghafte Änderung der Potentialverteilung auf, die mit einer Frequenz bis zu $10^{9} \mathrm{~Hz}$ erfolgen kann. Für dieses Bild sprechen die wenig unterschiedlichen relativen Häufigkeiten der einzelnen Dissoziationsprozesse in der Wechselstromentladung und die Bevorzugung eines bestimmten Trennungsprozesses in der Gleichstromentladung.

Die im Reaktionsraum vorhandenen Feldstärken von 20 - 40 Volt $/ \mathrm{cm}$ reichen demnach aus, das Cyclohexanmolekül im angeregten Zustand zu beeinflussen.

Für die Anregung zu diesen Untersuchungen und für eingehende Diskussionen bin ich dem verstorbenen Leiter der Forschungsstelle für Spektroskopie in der MaxPlanck-Gesellschaft, Herrn Professor Dr. H. SchüLER, zu Dank verpflichtet. 